\title{
An Efficient Approach for Solving Nonlinear Troesch's and Bratu's Problems by Wavelet Analysis Method
}

\author{
A. Kazemi Nasab, Z. Pashazadeh Atabakan, and A. Kılıçman \\ Department of Mathematics and Mathematical Research Institute, University Putra Malaysia, 43400 Serdang, Selangor, Malaysia \\ Correspondence should be addressed to A. K1lıçman; kilicman@yahoo.com
}

Received 10 June 2013; Accepted 22 July 2013

Academic Editor: Hossein Jafari

Copyright ( 2013 A. Kazemi Nasab et al. This is an open access article distributed under the Creative Commons Attribution License, which permits unrestricted use, distribution, and reproduction in any medium, provided the original work is properly cited.

\begin{abstract}
We introduce Chebyshev wavelet analysis method to solve the nonlinear Troesch and Bratu problems. Chebyshev wavelets expansions together with operational matrix of derivative are employed to reduce the computation of nonlinear problems to a system of algebraic equations. Several examples are given to validate the efficiency and accuracy of the proposed technique. We compare the results with those ones reported in the literature in order to demonstrate that the method converges rapidly and approximates the exact solution very accurately by using only a small number of Chebyshev wavelet basis functions. Convergence analysis is also included.
\end{abstract}

\section{Introduction}

Nonlinear equations occurring in a wide variety of problems in engineering and science have received a great deal of attention in the recent decades. Consider the Troesch nonlinear two-point boundary value problem [1-3] in the following form:

$$
\begin{gathered}
u^{\prime \prime}(t)+\lambda \sinh (\lambda u(t))=0, \quad t \in[0,1], \\
u(0)=0, \quad u(1)=1,
\end{gathered}
$$

where $\lambda$ is a positive constant. Troesch's problem comes from the investigation of the confinement of a plasma column under radiation pressure. Many authors have paid considerable attention to solving Troesch's problem. Weibel was the first to explain and solve the problem [4]. Roberts and Shipman [3] obtained the closed form solution of the problem in terms of the Jacobi elliptic function. Troesch obtained numerical solution of this problem by the shooting method [5]. Deeba et al. [6] proposed a numerical method based on the decomposition technique. A numerical scheme based on the modified homotopy perturbation method is deduced by Feng et al. [7]. Variational iteration method was proposed by Momani et al. [8]. Khuri solved this problem numerically based on Laplace transform and a modified decomposition technique [9] and also proposed B-spline collocation approach for solving Troesch's problem [10]. The Sinc-Galerkin method was introduced by Zarebnia and Sajjadian [11]. S.-H. Chang and I.-L. Chang [12] proposed a new technique based on differential transform for solving Troesch's problem. A new algorithm based on the variational method and variable transformation was proposed by Chang [13]. More detailed information and references to other discussions on Troesch's problem may be found in [14-18].

The closed form solution of Troesch's problem is given in [3], where $u^{\prime}(0)$ is the derivative at 0 given by

$$
u^{\prime}(0)=2(1-m)^{1 / 2} .
$$

The constant $m$ satisfies the transcendental equation

$$
\frac{\sinh (\lambda / 2)}{(1-m)^{1 / 2}}=s c(\lambda m),
$$

where $\operatorname{sc}(\lambda \mid m)$ is the Jacobi function defined by $\operatorname{sc}(\lambda \mid m)=$ $\sin \phi / \cos \phi$, where $\phi, \lambda$, and $m$ satisfy the following integral:

$$
\lambda=\int_{0}^{\phi} \frac{1}{\sqrt{1-m \sin ^{2} \theta}} d \theta .
$$


It has been indicated in $[3,5]$ that $u(t)$ has a singularity located approximately at

$$
t_{s}=\frac{1}{2 \lambda} \ln \left(\frac{16}{1-m}\right)=\frac{1}{\lambda} \ln \left(\frac{8}{u^{\prime}(0)}\right),
$$

which implies that the singularity lies within the integration range if $u^{\prime}(0)>8 e^{\lambda}$. This characteristic of Troesch's problem makes it impossible to be solved by some methods when $\lambda>$ $1[6,7,9,19]$.

Another well-known problem is the Bratu nonlinear twopoint boundary value problem given in the following form:

$$
\begin{gathered}
u^{\prime \prime}(t)+\lambda e^{u(t)}=0, \quad t \in(0,1), \\
u(0)=0, \quad u(1)=0,
\end{gathered}
$$

where $\lambda$ is a physical parameter and the prime denotes the differentiation with respect to $t$. The Bratu problem is indeed a special case of the following well-known classical Gelfand problem by assuming $N=1$ :

$$
\begin{gathered}
u^{\prime \prime}(t)+\frac{N-1}{r} u^{\prime}(t)+\lambda e^{u(t)}=0, \\
r \in(0,1], \quad N=1,2,3, \ldots, \\
u(0)=0, \quad u(1)=0,
\end{gathered}
$$

where $N=1,2$, and 3 correspond to the infinite slab, infinite circular cylinder, and sphere, respectively. The numerical solutions for all $N=1,2,3, \ldots$ were obtained by Joseph and Lundgren [20] for the domain of a unit ball.

The Bratu problem has an analytical solution given in the following form:

$$
u(t)=-2 \ln \left[\frac{\cosh ((t-(1 / 2))(\theta / 2))}{\cosh (\theta / 4)}\right],
$$

where $\theta$ is the solution of $\theta=\sqrt{2 \lambda} \cosh (\theta / 4)$.

The Bratu problem has zero, one, or two solutions when $\lambda>\lambda_{c}, \lambda=\lambda_{c}$, and $\lambda<\lambda_{c}$, respectively, where the critical value $\lambda_{c}$ satisfies the equation $1=(1 / 4) \sqrt{2 \lambda_{c}} \sinh \left(\theta_{c} / 4\right)$ and it was obtained in $[21,22]$ that the critical value $\lambda_{c}$ is given by $\lambda_{c}=3.513830719$.

The Bratu problem is used in a different variety of applications such as the fuel ignition of the thermal combustion theory, the model thermal reaction process, the Chandrasekhar model of the expansion of the universe, chemical reaction theory, radiative heat transfer, and nanotechnology [23-29].

Solving the Bratu Problem by analytical and numerical methods has gained considerable attention from many authors. Aregbesola applied weighted residual method [22] and Wazwaz has employed the Adomian decomposition method to get exact solutions [23]. Homotopy analysis method was developed by Liao and Tan [29] and Laplace transform decomposition method was used in [30]. Nonpolynomial spline method has been applied by Jalilian to obtain smooth approximate solution of the one-dimensional Bratu problem [31]. Caglar et al. [32] developed the Bspline method. Variational method and differential transform method were used in [33,34]. Also Abbasbandy et al. [35] tried to solve the problem using the Lie-group shooting method.

In the recent years, wavelets have received considerable attention by researchers in different fields of science and engineering. One advantage of wavelet analysis is the ability to perform local analysis [36]. Wavelet analysis is able to reveal signal aspects that other analysis methods miss, such as trends, breakdown points, and discontinuities. In comparison with other orthogonal functions, multiresolution analysis aspect of wavelets permits the accurate representation of a variety of functions and operators. In other words, we can change $M$ and $k$ simultaneously to get more accurate solution. In addition, the coefficient matrix of algebric equations obtained after dicretization is sparse. So it is computationally efficient to use wavelet methods for solving equations. In addition, the solution is convergent.

We organize our paper as follows. In Section 2, we introduce the Chebyshev wavelets, and the operational matrix of derivative for Chebyshev wavelets is defined. In Section 3, convergence analysis is included. In Section 4, we introduce the method of solving Troesch's and Bratu's problems by wavelet analysis method. Several numerical examples are included in Section 5 to confirm that our method is efficient and accurate. Some conclusions are drawn in Section 6.

\section{Chebyshev Wavelets and Their Properties}

2.1. Wavelets and Chebyshev Wavelets. Wavelets have been very successfully used in many scientific and engineering fields. They constitute a family of functions constructed from dilation and transformation of a single function called the mother wavelet $\psi(t)$; we have the following family of continuous wavelets:

$$
\psi_{a, b}(t)=|a|^{-1 / 2} \psi\left(\frac{t-b}{a}\right), \quad a, b \in R, a \neq 0 .
$$

Chebyshev wavelets, $\psi_{n, m}=\psi(k, n, m, t)$, have four arguments, $n=0,1, \ldots, 2^{k}-1$, where $k$ can assume any positive integer, $m$ is the degree of Chebyshev polynomials of the first kind, and $t$ denotes the time.

Consider

$$
\psi_{n, m}(t)= \begin{cases}2^{(k+1) / 2} \widetilde{T}_{m}\left(2^{k+1} t-2 n-1\right), & \frac{n}{2^{k}} \leq t<\frac{n+1}{2^{k}}, \\ 0, & \text { otherwise }\end{cases}
$$

where

$$
\widetilde{T}_{m}(t)= \begin{cases}\frac{1}{\sqrt{\pi}}, & m=0 \\ \sqrt{\frac{2}{\pi}} T_{m}(t), & m \geq 1\end{cases}
$$


and $m=0,1, \ldots, M$, and $n=0,1, \ldots, 2^{k}-1$. In (10) the coefficients are used for orthonormality. Here $T_{m}(t)$ are Chebyshev polynomials of the first kind of degree $m$ which are orthogonal with respect to the weight function, $w(t)=$ $1 / \sqrt{1-t^{2}}$, and satisfy the following recursive formula:

$$
\begin{gathered}
T_{0}(t)=1, \quad T_{1}(t)=t, \\
T_{m+1}(t)=2 t T_{m}(t)-T_{m-1}(t), \quad m=1,2, \ldots .
\end{gathered}
$$

Note that, in dealing with Chebyshev wavelets, the weight function $w(t)$ has to be dilated and translated as follows:

$$
w_{n, k}(t)=w\left(2^{k+1} t-2 n-1\right)
$$

in order to get orthogonal wavelets.

2.2. Function Approximation. A function $u(t) \in L_{\widetilde{w}}^{2}[0,1]$ (where $\widetilde{w}(t)=w(2 t-1)$ ) may be expanded as

$$
u(t)=\sum_{n=0}^{\infty} \sum_{m=0}^{\infty} c_{n, m} \psi_{n, m}(t)
$$

where $c_{n, m}=\left(u(t), \psi_{n, m}(t)\right)_{w_{n, k}}$, in which (.,.) denotes the inner product in $L_{\widetilde{w}}^{2}[0,1]$. If we consider truncated series in (15), we obtain

$$
u(t)=\sum_{n=0}^{2^{k}-1} \sum_{m=0}^{M} c_{n, m} \psi_{n, m}(t)=C^{T} \Psi(t)
$$

where $C$ and $\Psi(t)$ are $2^{k}(M+1) \times 1$ matrices given by

$$
\begin{gathered}
C=\left[c_{0,0}, c_{0,1}, \ldots, c_{0, M}, c_{1,0}, c_{1,1}, \ldots, c_{1, M}, \ldots,\right. \\
\left.c_{2^{k}-1,0}, c_{2^{k}-1,1}, \ldots, c_{2^{k}-1, M}\right]^{T}, \\
\Psi(t)=\left[\psi_{0,0}, \psi_{0,1}, \ldots, \psi_{0, M}, \psi_{1,0}, \psi_{1,1}, \ldots, \psi_{1, M}, \ldots,\right. \\
\left.\psi_{2^{k}-1,0}, \psi_{2^{k}-1,1}, \ldots, \psi_{2^{k}-1, M}\right]^{T} .
\end{gathered}
$$

2.3. Operational Matrix of Derivative (OMD). The derivative of the vector $\Psi(t)$, which is defined in (17), can be expressed by

$$
\frac{d}{d t} \psi(t)=D \psi(t)
$$

where $D$ is $2^{k}(M+1) \times 2^{k}(M+1)$ operational matrix of derivative defined as follows:

$$
D=\left[\begin{array}{cccc}
E & 0 & \cdots & 0 \\
0 & E & \cdots & 0 \\
\vdots & \vdots & \ddots & \vdots \\
0 & 0 & 0 & E
\end{array}\right],
$$

in which $E$ is $(M+1) \times(M+1)$ matrix and its $(i, j)$ th element is defined as follows:

$$
\begin{aligned}
& E_{i, j} \\
& = \begin{cases}2^{k+2} \sqrt[m]{\frac{\gamma_{i-1}}{\gamma_{j-1}},} \begin{array}{l}
i=2, \ldots,(M+1), j=1, \ldots, j-1 ; \\
(i+j) \text { is odd, } \\
0,
\end{array} & \text { otherwise, }\end{cases}
\end{aligned}
$$

where

$$
\gamma_{n}= \begin{cases}2, & n=0 \\ 1, & n \geq 1\end{cases}
$$

The method of calculation of $D$ is illustrated in [37].

Corollary 1 . The operational matrix for nth derivative can be obtained using (18) as

$$
\frac{d^{n} \Psi(t)}{d x^{n}}=D^{n} \Psi(t)
$$

where $D^{n}$ is the nth power of matrix $D$.

\section{Convergence Analysis}

Lemma 2. If the Chebyshev wavelet expansion of a continuous function $u(t)$ converges uniformly, then the Chebyshev wavelet expansion converges to the function $u(t)$.

Proof. Suppose the Chebyshev wavelet expansion of the continuous function $u(t)$ converges to function $v(t)$

$$
v(t)=\sum_{n=1}^{2^{k-1}} \sum_{m=0}^{M} \widehat{c}_{n m} \psi_{n m}(t)
$$

where $\widehat{c}_{n, m}=\left(u(t), \psi_{n, m}(t)\right)_{w_{n, k}}$. Multiply both sides of (23) by $\psi_{r, s}(t) w_{r, k}(t)$, where $r$ and $s$ are fixed. Due to the uniform convergence, we can then integrate termwise on interval $[0,1]$

$$
\begin{aligned}
\int_{0}^{1} v(t) \psi_{r, s}(t) w_{r, k} d t \\
\quad=\sum_{n=1}^{2^{k-1}} \sum_{m=0}^{M} \int_{0}^{1} \widehat{c}_{n m} \psi_{n m}(t) \psi_{r, s}(t) w_{r, k} d t .
\end{aligned}
$$

By the orthonormality of wavelet basis functions, we have

$$
\int_{0}^{1} v(t) \psi_{r, s}(t) w_{r, k} d t=\widehat{c}_{r, s} .
$$

Thus $\left(v(t), \psi_{n, m}(t)\right)_{w_{n, k}}=\widehat{c}_{n, m}$ for $n=1,2, \ldots, 2^{k-1}$ and $m=$ $0,1, \ldots, M$; consequently, $u(t)$ and $v(t)$ have the same Fourier expansions with Chebyshev wavelet basis; therefore, $u(t)=$ $v(t)$, for $t \in[0,1][38]$.

Theorem 3. A function $u(t) \in L_{w_{n, k}}^{2}([0,1])$, with bounded second derivative, say $\left|u^{\prime \prime}(t)\right| \leq B$, can be expanded as 
TABLE 1: Obtained absolute errors of Troesch's problem for $\lambda=0.5$.

\begin{tabular}{lcccccc}
\hline$t$ & $\begin{array}{c}\text { Chebyshev wavelet } \\
M=4, k=1\end{array}$ & $\begin{array}{c}\text { Laplace } \\
{[9]}\end{array}$ & $\begin{array}{c}\text { HPM } \\
{[7]}\end{array}$ & $\begin{array}{c}\text { HAM } \\
{[18]}\end{array}$ & $\begin{array}{c}\text { Spline } \\
{[10]}\end{array}$ & $\begin{array}{c}\text { VIM } \\
{[7]}\end{array}$ \\
\hline 0.1 & $7.6 \times 10^{-4}$ & $7.7 \times 10^{-4}$ & $8.2 \times 10^{-4}$ & $7.7 \times 10^{-4}$ & $7.7 \times 10^{-4}$ & $4.9 \times 10^{-3}$ \\
0.2 & $1.5 \times 10^{-3}$ & $1.5 \times 10^{-3}$ & $1.6 \times 10^{-3}$ & $1.5 \times 10^{-3}$ & $1.5 \times 10^{-3}$ \\
0.3 & $2.1 \times 10^{-3}$ & $2.1 \times 10^{-3}$ & $2.3 \times 10^{-3}$ & $2.1 \times 10^{-3}$ & $2.1 \times 10^{-3}$ & $9.7 \times 10^{-3}$ \\
0.4 & $2.7 \times 10^{-3}$ & $2.7 \times 10^{-3}$ & $2.9 \times 10^{-3}$ & $2.7 \times 10^{-3}$ & $2.7 \times 10^{-3}$ & $1.9 \times 10^{-2}$ \\
0.5 & $3.0 \times 10^{-3}$ & $3.0 \times 10^{-3}$ & $3.2 \times 10^{-3}$ & $3.0 \times 10^{-3}$ & $3.0 \times 10^{-3}$ & $2.3 \times 10^{-2}$ \\
0.6 & $3.1 \times 10^{-3}$ & $3.1 \times 10^{-3}$ & $3.4 \times 10^{-3}$ & $3.1 \times 10^{-3}$ & $3.1 \times 10^{-3}$ & $2.8 \times 10^{-2}$ \\
0.7 & $3.0 \times 10^{-3}$ & $3.0 \times 10^{-3}$ & $3.2 \times 10^{-3}$ & $3.0 \times 10^{-3}$ & $3.0 \times 10^{-3}$ & $3.2 \times 10^{-2}$ \\
0.8 & $2.4 \times 10^{-3}$ & $2.4 \times 10^{-3}$ & $2.7 \times 10^{-3}$ & $2.4 \times 10^{-3}$ & $2.4 \times 10^{-3}$ \\
0.9 & $1.5 \times 10^{-3}$ & $1.5 \times 10^{-3}$ & $1.6 \times 10^{-3}$ & $1.5 \times 10^{-3}$ & $3.6 \times 10^{-2}$ \\
& & & & & $4.0 \times 10^{-3}$ \\
\hline
\end{tabular}

TABLE 2: Obtained absolute errors of Troesch's problem for $\lambda=1.0$.

\begin{tabular}{lcccccc}
\hline$t$ & $\begin{array}{c}\text { Chebyshev wavelet } \\
M=4, k=1\end{array}$ & $\begin{array}{c}\text { Laplace } \\
{[9]}\end{array}$ & $\begin{array}{c}\text { HPM } \\
{[7]}\end{array}$ & $\begin{array}{c}\text { HAM } \\
{[18]}\end{array}$ & $\begin{array}{c}\text { Spline } \\
{[10]}\end{array}$ & $\begin{array}{c}\text { VIM } \\
{[7]}\end{array}$ \\
\hline 0.1 & $2.8 \times 10^{-3}$ & $2.9 \times 10^{-3}$ & $3.6 \times 10^{-3}$ & $2.9 \times 10^{-3}$ & $2.8 \times 10^{-3}$ \\
0.2 & $5.6 \times 10^{-3}$ & $5.9 \times 10^{-3}$ & $7.1 \times 10^{-2}$ & $5.7 \times 10^{-3}$ & $5.6 \times 10^{-3}$ \\
0.3 & $8.2 \times 10^{-3}$ & $8.2 \times 10^{-3}$ & $1.0 \times 10^{-2}$ & $8.3 \times 10^{-3}$ & $8.2 \times 10^{-3}$ & $3.6 \times 10^{-2}$ \\
0.4 & $1.0 \times 10^{-2}$ & $1.0 \times 10^{-2}$ & $1.3 \times 10^{-2}$ & $1.0 \times 10^{-2}$ & $1.0 \times 10^{-2}$ \\
0.5 & $1.2 \times 10^{-2}$ & $1.2 \times 10^{-2}$ & $1.6 \times 10^{-2}$ & $1.2 \times 10^{-2}$ & $1.2 \times 10^{-2}$ \\
0.6 & $1.3 \times 10^{-2}$ & $1.3 \times 10^{-2}$ & $1.7 \times 10^{-2}$ & $1.3 \times 10^{-2}$ & $1.3 \times 10^{-2}$ \\
0.7 & $1.3 \times 10^{-2}$ & $1.3 \times 10^{-2}$ & $1.7 \times 10^{-2}$ & $1.2 \times 10^{-2}$ & $1.3 \times 10^{-2}$ \\
0.8 & $1.1 \times 10^{-2}$ & $1.1 \times 10^{-2}$ & $1.5 \times 10^{-2}$ & $1.2 \times 10^{-2}$ & $1.3 \times 10^{-1}$ \\
0.9 & $7.4 \times 10^{-3}$ & $7.4 \times 10^{-3}$ & $9.7 \times 10^{-3}$ & $7.4 \times 10^{-3}$ & $1.1 \times 10^{-2}$ & $7.4 \times 10^{-3}$ \\
\hline
\end{tabular}

an infinite sum of Chebyshev wavelets, and the series converges uniformly to $u(t)$; that is,

$$
u(t)=\sum_{n=1}^{\infty} \sum_{m=0}^{\infty} \widehat{c}_{n m} \psi_{n m}(t)
$$

Proof. We have

$$
\begin{aligned}
\widehat{c}_{n, m}= & \left(u(t), \psi_{n, m}(t)\right)_{w_{n, k}} \\
= & \int_{0}^{1} u(t) \psi_{n m}(t) w_{n, k}(t) d t \\
= & \int_{n-1 / 2^{k-1}}^{n / 2^{k-1}} 2^{k / 2} p_{m} u(t) T_{m}\left(2^{k} t-2 n+1\right) \\
& \times w\left(2^{k} t-2 n+1\right) d t .
\end{aligned}
$$

For $m>1$, by substituting $2^{k} t-2 n+1=\cos \alpha$, it yields

$$
\widehat{c}_{n m}=\frac{1}{2^{k / 2}} \int_{0}^{\pi} u\left(\frac{\cos \alpha+2 n-1}{2^{k}}\right) \sqrt{\frac{2}{\pi}} \cos m \alpha d \alpha .
$$

Using integration by parts, we get

$$
\begin{aligned}
\widehat{c}_{n m}= & \left.\frac{\sqrt{2}}{2^{k / 2} \sqrt{\pi}} u\left(\frac{\cos \alpha+2 n-1}{2^{k}}\right)\left(\frac{\sin m \alpha}{m}\right)\right|_{0} ^{\pi} \\
& +\frac{\sqrt{2}}{2^{3 k / 2} m \sqrt{\pi}} \int_{0}^{\pi} u^{\prime}\left(\frac{\cos \alpha+2 n-1}{2^{k}}\right) \sin m \alpha \sin \alpha d \alpha .
\end{aligned}
$$

The first part is zero; therefore,

$$
\widehat{c}_{n m}=\frac{\sqrt{2}}{2^{3 k / 2} m \sqrt{\pi}} \int_{0}^{\pi} u^{\prime}\left(\frac{\cos \alpha+2 n-1}{2^{k}}\right) \sin m \alpha \sin \alpha d \alpha .
$$

Using integration by parts again, it yields

$$
\begin{aligned}
\widehat{c}_{n m}= & \frac{1}{2^{3 k / 2} m \sqrt{2 \pi}} \\
& \times\left. u^{\prime}\left(\frac{\cos \alpha+2 n-1}{2^{k}}\right)\left(\frac{\sin (m-1) \alpha}{m-1}-\frac{\sin (m+1) \alpha}{m+1}\right)\right|_{0} ^{\pi} \\
& +\frac{1}{2^{5 k / 2} m \sqrt{2 \pi}} \int_{0}^{\pi} u^{\prime \prime}\left(\left(\frac{\cos \alpha+2 n-1}{2^{k}}\right) r_{m}(\alpha)\right) d \alpha,
\end{aligned}
$$

where

$$
r_{m}(\alpha)=\sin \alpha\left(\frac{\sin (m-1) \alpha}{m-1}-\frac{\sin (m+1) \alpha}{m+1}\right) .
$$


TABLE 3: Numerical solution of Troesch's problem for $\lambda=5$.

\begin{tabular}{lccccc}
\hline$t$ & Fortran code & $\begin{array}{c}\text { B-spline } \\
{[10]}\end{array}$ & $\mid$ Error & \multicolumn{2}{c}{$\begin{array}{c}\text { Chebyshev wavelet } \\
M=9, k=3\end{array}$} \\
\hline 0.0 & 0.00000000 & 0.00000000 & 0.0 & 0.00000000 & 0.0 \\
0.2 & 0.01075342 & 0.01002027 & $7.3 \times 10^{-3}$ & 0.01075412 & $7.0 \times 10^{-7}$ \\
0.4 & 0.03320051 & 0.03099793 & $2.2 \times 10^{-3}$ & 0.03320271 & $2.2 \times 10^{-6}$ \\
0.8 & 0.25821664 & 0.24170496 & $1.4 \times 10^{-2}$ & 0.25823492 & $1.8 \times 10^{-5}$ \\
0.9 & 0.45506034 & 0.42461830 & $3.0 \times 10^{-2}$ & 0.45508401 & $2.4 \times 10^{-5}$ \\
1.0 & 1.00000000 & 1.00000000 & 0.0 & 1.00000000 & 0.0 \\
\hline
\end{tabular}

Thus, we get

$$
\begin{aligned}
\left|\widehat{c}_{n m}\right| & =\left|\frac{1}{2^{5 k / 2} m \sqrt{2 \pi}} \int_{0}^{\pi} u^{\prime \prime}\left(\frac{\cos \alpha+2 n-1}{2^{k}}\right) r_{m}(\alpha) d \alpha\right| \\
& \leq\left(\frac{1}{2^{5 k / 2} m \sqrt{2 \pi}}\right) \int_{0}^{\pi}\left|u^{\prime \prime}\left(\frac{\cos \alpha+2 n-1}{2^{k}}\right) r_{m}(\alpha)\right| d \alpha \\
& \leq \frac{B}{2^{5 k / 2} m \sqrt{2 \pi}} \int_{0}^{\pi}\left|r_{m}(\alpha)\right| d \alpha .
\end{aligned}
$$

However

$$
\begin{aligned}
& \int_{0}^{\pi}\left|r_{m}(\alpha)\right| d \alpha \\
& \quad=\int_{0}^{\pi}\left|\sin \alpha\left(\frac{\sin (m-1) \alpha}{m-1}-\frac{\sin (m+1) \alpha}{m+1}\right)\right| d \alpha \\
& \quad \leq \int_{0}^{\pi}\left|\frac{\sin \alpha \sin (m-1) \alpha}{m-1}\right|+\left|\frac{\sin \alpha \sin (m+1) \alpha}{m+1}\right| d \alpha \\
& \quad \leq \frac{2 m \pi}{m^{2}-1} .
\end{aligned}
$$

Since $n \leq 2^{k-1}$, we obtain

$$
\left|\widehat{c}_{n m}\right| \leq \frac{\sqrt{2 \pi} B}{(2 n)^{5 / 2}\left(m^{2}-1\right)} .
$$

Now, if $m=1$, by using (30), we have

$$
\left|\widehat{c}_{n 1}\right|<\frac{\sqrt{2 \pi}}{(2 n)^{3 / 2}} \max _{0 \leq t \leq 1}\left|u^{\prime}(t)\right| .
$$

It is mentioned in [39] that $\left\{\psi_{n 0}\right\}_{n=1}^{\infty}$ form an orthogonal system constructed by Haar scaling function with respect to the weight function $w(t)$, and so $\sum_{n=1}^{\infty} \widehat{c}_{n 0} \psi_{n 0}(t)$ is convergent. Hence, we will have

$$
\begin{aligned}
& \left|\sum_{n=1}^{\infty} \sum_{m=0}^{\infty} \widehat{c}_{n m} \psi_{n m}(t)\right| \\
& \leq\left|\sum_{n=1}^{\infty} \widehat{c}_{n 0} \psi_{n 0}(t)\right|+\sum_{n=1}^{\infty} \sum_{m=1}^{\infty}\left|\widehat{c}_{n m}\right|\left|\psi_{n m}(t)\right| \\
& \leq\left|\sum_{n=1}^{\infty} \widehat{c}_{n 0} \psi_{n 0}(t)\right|+\sum_{n=1}^{\infty} \sum_{m=1}^{\infty}\left|\widehat{c}_{n m}\right|<\infty .
\end{aligned}
$$

Therefore, in view of Lemma 2, the series $\sum_{n=1}^{\infty} \sum_{m=1}^{\infty} \widehat{c}_{n m} \psi_{n m}(t)$ converges to $u(t)$ uniformly.

\section{The Chebyshev Wavelet Analysis Method}

4.1. Troesch's Problem. Troesch's problems (1) can be considered as follows:

$$
\begin{gathered}
u^{\prime}=w, \\
w^{\prime}=\lambda \sinh (\lambda u), \\
u(0)=0, \quad u(1)=1 .
\end{gathered}
$$

Then the Jacobian matrix of system (38) is given by

$$
J(u, w)=\left[\begin{array}{cc}
0 & 1 \\
\lambda^{2} \cosh (\lambda u) & 0
\end{array}\right]
$$

Therefore, the eigenvalues of the Jacobian matrix at the end points of the interval $[0,1]$ are

$$
\lambda^{\prime}(0)= \pm \lambda, \quad \lambda^{\prime}(1)= \pm \lambda \sqrt{\cosh (\lambda)} .
$$

If we choose large $\lambda$, we will have large eigenvalues; for example, if $\lambda=10$, then the eigenvalue becomes $\lambda^{\prime}= \pm 1049$. On the other hand, the Jacobian matrix $J$ is normal if and only if $\lambda^{2} \cosh (\lambda u)=1$. This equality is satisfied only for relatively small values of $\lambda$. This clarifies why conventional methods, such as finite differences, are not convenient for large values of $\lambda[10]$. To solve Troesch's problems where there exist, a strong nonlinear term $\sinh (\lambda u)$ and boundary layer only at the right endpoint $(\lambda>1)$ [10], we propose Chebyshev wavelet analysis method especially to handle larger eigenvalues.

Consider the Troesch nonlinear boundary value problem

$$
u^{\prime \prime}(t)+\lambda \sinh (\lambda u(t))=0, \quad t \in(0,1),
$$

with the boundary conditions

$$
u(0)=0, \quad u(1)=1 .
$$

In order to solve the problem, we first approximate all functions $u(t)$ and $u^{\prime \prime}(t)$ using basis functions $\Psi(t)$ as

$$
u(t)=C^{T} \Psi(t), \quad u^{\prime \prime}(t)=C^{T} D^{2} \Psi(t) .
$$

Substituting (43) in (41), we obtain

$$
C^{T} D^{2} \Psi(t)-\lambda \sinh \left(\lambda C^{T} \Psi(t)\right)=0 .
$$


TABLE 4: Numerical solution of Troesch's problem for $\lambda=10$.

\begin{tabular}{|c|c|c|c|c|c|}
\hline$t$ & $\begin{array}{l}\text { Present method } \\
M=20, k=5\end{array}$ & $\begin{array}{c}\text { B-spline, } N=790 \\
{[10]}\end{array}$ & $\begin{array}{c}y_{1750}(x) \\
{[12]}\end{array}$ & $\begin{array}{c}y_{1}(x)=\frac{4}{\mu} \tanh ^{-1}\left(\mu_{1}(x)\right) \\
{[13]}\end{array}$ & $\begin{array}{c}y_{2}(x)=\frac{4}{\mu} \tanh ^{-1}\left(\mu_{2}(x)\right) \\
{[13]}\end{array}$ \\
\hline 0.00 & 0 & 0 & 0 & 0 & 0 \\
\hline 0.100 & $4.2110 \times 10^{-5}$ & $4.2097 \times 10^{-5}$ & $4.2113 \times 10^{-5}$ & $4.2112 \times 10^{-5}$ & $4.2112 \times 10^{-5}$ \\
\hline 0.200 & $1.2996 \times 10^{-4}$ & $1.2992 \times 10^{-4}$ & $1.2997 \times 10^{-4}$ & $1.2996 \times 10^{-4}$ & $1.2996 \times 10^{-4}$ \\
\hline 0.300 & $3.5896 \times 10^{-4}$ & $3.5886 \times 10^{-4}$ & $3.5899 \times 10^{-4}$ & $3.5898 \times 10^{-4}$ & $3.5898 \times 10^{-4}$ \\
\hline 0.400 & $9.7785 \times 10^{-4}$ & $9.7762 \times 10^{-4}$ & $9.7792 \times 10^{-4}$ & $9.7790 \times 10^{-4}$ & $9.7790 \times 10^{-4}$ \\
\hline 0.500 & $2.6589 \times 10^{-3}$ & $2.6583 \times 10^{-3}$ & $2.6591 \times 10^{-3}$ & $2.6590 \times 10^{-3}$ & $2.6590 \times 10^{-3}$ \\
\hline 0.600 & $7.2286 \times 10^{-3}$ & $7.2272 \times 10^{-3}$ & $7.2291 \times 10^{-3}$ & $7.2289 \times 10^{-3}$ & $7.2289 \times 10^{-3}$ \\
\hline 0.700 & $1.9663 \times 10^{-2}$ & $1.9660 \times 10^{-2}$ & $1.9664 \times 10^{-2}$ & $1.9664 \times 10^{-2}$ & $1.9664 \times 10^{-2}$ \\
\hline 0.800 & $5.3728 \times 10^{-2}$ & $5.3720 \times 10^{-2}$ & $5.3732 \times 10^{-2}$ & $5.3730 \times 10^{-2}$ & $5.3730 \times 10^{-2}$ \\
\hline 0.900 & $1.5210 \times 10^{-1}$ & $1.5209 \times 10^{-1}$ & $1.5212 \times 10^{-1}$ & $1.5211 \times 10^{-1}$ & $1.5211 \times 10^{-1}$ \\
\hline 0.925 & $2.0199 \times 10^{-1}$ & $2.0199 \times 10^{-1}$ & $2.0201 \times 10^{-1}$ & $2.0201 \times 10^{-1}$ & $2.0201 \times 10^{-1}$ \\
\hline 0.950 & $2.7625 \times 10^{-1}$ & $2.7623 \times 10^{-1}$ & $2.7628 \times 10^{-1}$ & $2.7627 \times 10^{-1}$ & $2.7627 \times 10^{-1}$ \\
\hline 0.970 & $3.7224 \times 10^{-1}$ & $3.7223 \times 10^{-1}$ & $3.7229 \times 10^{-1}$ & $3.7226 \times 10^{-1}$ & $3.7226 \times 10^{-1}$ \\
\hline 0.980 & $4.4822 \times 10^{-1}$ & $4.4820 \times 10^{-1}$ & $4.4825 \times 10^{-1}$ & $4.4823 \times 10^{-1}$ & $4.4823 \times 10^{-1}$ \\
\hline 0.990 & $5.7407 \times 10^{-1}$ & $5.7405 \times 10^{-1}$ & $5.7411 \times 10^{-1}$ & $5.7408 \times 10^{-1}$ & $5.7408 \times 10^{-1}$ \\
\hline 0.995 & $6.9011 \times 10^{-1}$ & $6.9010 \times 10^{-1}$ & $6.9018 \times 10^{-1}$ & $6.9011 \times 10^{-1}$ & $6.9011 \times 10^{-1}$ \\
\hline 0.997 & $7.6577 \times 10^{-1}$ & $7.6576 \times 10^{-1}$ & $7.6587 \times 10^{-1}$ & $7.6577 \times 10^{-1}$ & $7.6577 \times 10^{-1}$ \\
\hline 0.998 & $8.1802 \times 10^{-1}$ & $8.1802 \times 10^{-1}$ & $8.1816 \times 10^{-1}$ & $8.1803 \times 10^{-1}$ & $8.1803 \times 10^{-1}$ \\
\hline 0.999 & $8.8899 \times 10^{-1}$ & $8.8899 \times 10^{-1}$ & $8.8917 \times 10^{-1}$ & $8.8899 \times 10^{-1}$ & $8.8899 \times 10^{-1}$ \\
\hline 1.000 & 1.0000 & 1.0000 & 1.0000 & 9.9999 & 9.9999 \\
\hline
\end{tabular}

Now, we have $2^{k}(M+1)-2$ nonlinear equations by collocating (41) at $2^{k}(M+1)-2$ suitable collocation, points. From boundary conditions we also get two equations. So we have a nonlinear system of $2^{k}(M+1)$ equations with the same number of unknowns which can be solved by Newton's iterative method to obtain the vector $C$ and consequently the approximated solution $u(t)$.

4.2. Bratu's Problem. Consider the Bratu nonlinear boundary value problem

$$
u^{\prime \prime}(t)+\lambda e^{u(t)}=0, \quad t \in(0,1),
$$

with the boundary conditions

$$
u(0)=0, \quad u(1)=0,
$$

or initial conditions

$$
u(0)=0, \quad u^{\prime}(0)=0 .
$$

In order to solve the problem, we first approximate all functions $u(t), u^{\prime}(t)$, and $u^{\prime \prime}(t)$ using basis functions $\Psi(t)$ as

$$
\begin{gathered}
u(t)=C^{T} \Psi(t), \\
u^{\prime}(t)=C^{T} D \Psi(t), \quad u^{\prime \prime}(t)=C^{T} D^{2} \Psi(t) .
\end{gathered}
$$

Substituting (48) in (45), we obtain

$$
C^{T} D^{2} \Psi(t)+\lambda e^{C^{T} \Psi(t)}=0 .
$$

Now, we have $2^{k}(M+1)-2$ nonlinear equations by collocating (49) at $2^{k}(M+1)-2$ suitable collocation points. From boundary conditions, or initial conditions, we also get two equations. So we have a nonlinear system of $2^{k}(M+1)$ equations with the same number of unknowns which can be solved by Newton's iterative method to obtain the vector $C$ and consequently the approximated solution $u(t)$.

\section{Numerical Examples}

In this section, we solve Troesch's and Bratu's problems for different values of the parameter $\lambda$ using the computer algebra system Maple and make a comparison between our results and those ones reported in the literature to confirm the efficiency and accuracy of our method.

Example 1. Troesch's problem for $\lambda=0.5,1$, and 10 .

In Tables 1 and 2 , the absolute errors in solutions obtained by the introduced method for $\lambda=0.5$ and $\lambda=1$, respectively, are compared with those ones reported by other existing methods. We observe that the wavelet analysis method with only a few number of basis functions is comparable to Laplace, HAM, and spline methods but is slightly better than perturbation method and much better than variational method in terms of accuracy.

Due to the nonlinear term $\sinh (\lambda u(t))$, which is not analytic, some methods like Laplace, variational iteration method, and homotopy are not able to solve the Troesch problem when $\lambda \geq 5$. In Table 3 , the numerical solution for 
TABLE 5: Obtained absolute errors for $\lambda=1$.

\begin{tabular}{lcccccc}
\hline$t$ & $\begin{array}{c}\text { Present method } \\
M=10, k=3\end{array}$ & $\begin{array}{c}\text { NPSM } \\
{[31]}\end{array}$ & $\begin{array}{c}\text { LGSM } \\
{[35]}\end{array}$ & $\begin{array}{c}\text { Decomposition } \\
{[6]}\end{array}$ & $\begin{array}{c}\text { Laplace } \\
{[30]}\end{array}$ & $\begin{array}{c}\text { B-spline } \\
{[32]}\end{array}$ \\
\hline 0.1 & $1.23 \times 10^{-19}$ & $5.77 \times 10^{-10}$ & $7.51 \times 10^{-7}$ & $2.68 \times 10^{-3}$ & $1.98 \times 10^{-6}$ & $2.98 \times 10^{-6}$ \\
0.2 & $3.13 \times 10^{-19}$ & $2.47 \times 10^{-10}$ & $1.02 \times 10^{-6}$ & $2.02 \times 10^{-3}$ & $3.94 \times 10^{-6}$ & $5.46 \times 10^{-6}$ \\
0.3 & $6.20 \times 10^{-19}$ & $4.56 \times 10^{-11}$ & $9.05 \times 10^{-7}$ & $1.52 \times 10^{-4}$ & $5.85 \times 10^{-6}$ & $7.33 \times 10^{-6}$ \\
0.4 & $7.80 \times 10^{-19}$ & $9.64 \times 10^{-11}$ & $5.24 \times 10^{-7}$ & $2.20 \times 10^{-3}$ & $7.70 \times 10^{-6}$ & $8.50 \times 10^{-6}$ \\
0.5 & $7.70 \times 10^{-19}$ & $1.46 \times 10^{-10}$ & $5.07 \times 10^{-9}$ & $3.01 \times 10^{-3}$ & $9.47 \times 10^{-6}$ & $8.89 \times 10^{-6}$ \\
0.6 & $7.80 \times 10^{-19}$ & $9.64 \times 10^{-11}$ & $5.14 \times 10^{-7}$ & $2.20 \times 10^{-3}$ & $1.11 \times 10^{-5}$ & $8.50 \times 10^{-6}$ \\
0.7 & $6.20 \times 10^{-19}$ & $4.56 \times 10^{-11}$ & $8.95 \times 10^{-7}$ & $1.52 \times 10^{-4}$ & $1.26 \times 10^{-5}$ & $7.33 \times 10^{-6}$ \\
0.8 & $3.13 \times 10^{-19}$ & $2.47 \times 10^{-10}$ & $1.01 \times 10^{-6}$ & $2.02 \times 10^{-3}$ & $1.35 \times 10^{-5}$ & $5.46 \times 10^{-6}$ \\
0.9 & $1.22 \times 10^{-19}$ & $5.77 \times 10^{-10}$ & $7.42 \times 10^{-7}$ & $2.68 \times 10^{-3}$ & $1.20 \times 10^{-5}$ & $2.98 \times 10^{-6}$ \\
\hline
\end{tabular}

$\lambda=5$ obtained by the current method is compared with the numerical approximation of the exact solutions given by a Fortran code called TWPBVP and B-spline method [10]. It can be seen that our obtained results are much more accurate than those obtained by B-spline method.

In Table 4, the numerical solution obtained by the current method using $M=20$ and $k=5$ (672-term approximant), for $\lambda=10$, is compared with the results obtained by Bspline method over a nonuniform mesh using $n=790$ mesh points [10], with those in [12] computed using 1750term approximant, and with those obtained by [13] using a method based on the variational iteration method and variable transformation. It can be seen that the results obtained by the present method with much lesser number of terms to approximate the solution are compatible and in well agreement with those ones obtained by Chang [13].

Example 2. Consider the Bratu problem for $\lambda=1$.

We solve the problem by applying the technique described in Section 4 with $M=10$ and $k=3$. The absolute errors in solutions are tabulated in Table 5 . As can be seen in Table 5, only a small number of Chebyshev wavelet basis functions are needed to get the approximate solution which is in full agreement with the exact solution up to 18 digits while, using other methods, we can find a numerical approximation to the exact solution which is the same at most in 10 digits. We display the exact and obtained solutions in Figure 1. Absolute errors in solutions are plotted in Figure 2.

Example 3. Consider the Bratu problem for $\lambda=2$.

We solve the problem with $M=10$ and $k=3$. The absolute errors in solutions are tabulated in Table 6. We display the exact and obtained solutions in Figure 3. The plot of absolute errors in solutions in Figure 4 confirms the priority of our method over other methods in terms of efficiency and accuracy.

Example 4. Consider the Bratu problem for $\lambda=3.51$.

In this example, we set $M=10$ and $k=3$. The absolute errors in solutions are tabulated in Table 7. As can be seen in Table 7, when $\lambda$ is close to the critical value $\lambda_{c}$, some of the mentioned methods are not able to handle the problem

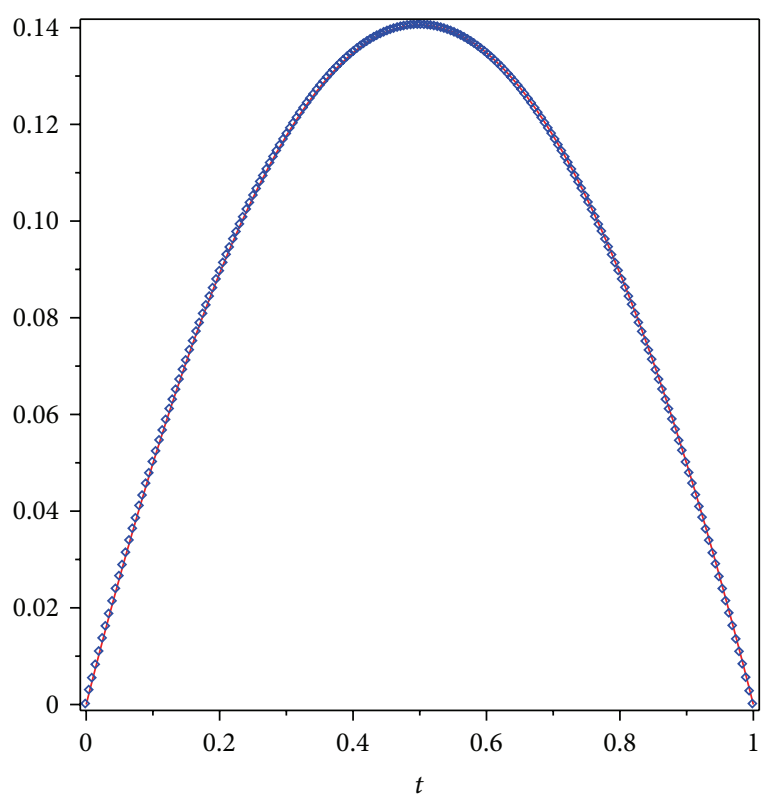

FIGURE 1: Plot of exact and approximated solutions for $\lambda=1$.

very well. However, using wavelet analysis method, we get the approximate solution which is much more accurate than nonpolynomial spline method and Lie-group shooting method. We display the exact and obtained solutions in Figure 5. Absolute errors in solutions are illustrated in Figure 6.

The maximum absolute errors in solutions for different values of $M, k$, and $\lambda$ are tabulated in Table 8 . According to Table 8, we can conclude that more accurate results can be obtained by increasing the values of $M$ and $k$ properly.

\section{Conclusion}

The well-known nonlinear Troesch and Bratu problems arise in a different variety of applications, and many researchers have drawn attention to solve them. The difficulty in this type of problems, due to existing strong nonlinear terms, is overcome here. The main characteristic of the proposed 
TABLE 6: Obtained absolute errors for $\lambda=2$.

\begin{tabular}{|c|c|c|c|c|c|c|}
\hline$t$ & $\begin{array}{c}\text { Present method } \\
M=10, k=3\end{array}$ & $\begin{array}{c}\text { NPSM } \\
{[31]} \\
\end{array}$ & $\begin{array}{c}\text { LGSM } \\
{[35]} \\
\end{array}$ & $\begin{array}{c}\text { Decomposition } \\
{[6]}\end{array}$ & $\begin{array}{c}\text { Laplace } \\
{[30]}\end{array}$ & $\begin{array}{c}\text { B-spline } \\
\text { [32] }\end{array}$ \\
\hline 0.1 & $2.03 \times 10^{-18}$ & $9.71 \times 10^{-9}$ & $4.03 \times 10^{-6}$ & $1.52 \times 10^{-2}$ & $2.13 \times 10^{-3}$ & $1.72 \times 10^{-5}$ \\
\hline 0.2 & $9.58 \times 10^{-18}$ & $1.41 \times 10^{-8}$ & $5.70 \times 10^{-6}$ & $1.47 \times 10^{-2}$ & $4.21 \times 10^{-3}$ & $3.26 \times 10^{-5}$ \\
\hline 0.3 & $2.86 \times 10^{-17}$ & $1.98 \times 10^{-8}$ & $5.22 \times 10^{-6}$ & $5.89 \times 10^{-3}$ & $6.19 \times 10^{-3}$ & $4.49 \times 10^{-5}$ \\
\hline 0.4 & $5.44 \times 10^{-17}$ & $2.42 \times 10^{-8}$ & $3.07 \times 10^{-6}$ & $3.25 \times 10^{-3}$ & $8.00 \times 10^{-3}$ & $5.28 \times 10^{-5}$ \\
\hline 0.5 & $6.87 \times 10^{-17}$ & $2.60 \times 10^{-8}$ & $1.46 \times 10^{-8}$ & $6.98 \times 10^{-3}$ & $9.60 \times 10^{-3}$ & $5.56 \times 10^{-5}$ \\
\hline 0.6 & $5.44 \times 10^{-17}$ & $2.42 \times 10^{-8}$ & $3.05 \times 10^{-6}$ & $3.25 \times 10^{-3}$ & $1.09 \times 10^{-3}$ & $5.28 \times 10^{-5}$ \\
\hline 0.7 & $2.86 \times 10^{-17}$ & $1.98 \times 10^{-8}$ & $5.19 \times 10^{-6}$ & $5.89 \times 10^{-3}$ & $1.19 \times 10^{-2}$ & $4.49 \times 10^{-5}$ \\
\hline 0.8 & $9.58 \times 10^{-18}$ & $1.41 \times 10^{-8}$ & $5.68 \times 10^{-6}$ & $1.47 \times 10^{-2}$ & $1.24 \times 10^{-2}$ & $3.26 \times 10^{-5}$ \\
\hline 0.9 & $2.03 \times 10^{-18}$ & $9.71 \times 10^{-9}$ & $4.01 \times 10^{-6}$ & $1.52 \times 10^{-2}$ & $1.09 \times 10^{-2}$ & $1.72 \times 10^{-5}$ \\
\hline
\end{tabular}

TABLE 7: Obtained absolute errors for $\lambda=3.51$.

\begin{tabular}{lcccr}
\hline$t$ & $\begin{array}{c}\text { Present method } \\
M=10, k=3\end{array}$ & $\begin{array}{c}\text { NPSM } \\
{[31]}\end{array}$ & $\begin{array}{c}\text { LGSM } \\
{[35]}\end{array}$ \\
\hline 0.1 & $2.34 \times 10^{-10}$ & $6.61 \times 10^{-6}$ & $4.45 \times 10^{-5}$ & $3.84 \times 10^{-2}$ \\
0.2 & $3.20 \times 10^{-10}$ & $5.83 \times 10^{-6}$ & $7.12 \times 10^{-5}$ & $7.48 \times 10^{-2}$ \\
0.3 & $7.88 \times 10^{-10}$ & $6.19 \times 10^{-6}$ & $7.30 \times 10^{-5}$ & $1.06 \times 10^{-1}$ \\
0.4 & $1.11 \times 10^{-9}$ & $6.89 \times 10^{-6}$ & $4.47 \times 10^{-5}$ & $1.27 \times 10^{-1}$ \\
0.5 & $1.22 \times 10^{-9}$ & $7.31 \times 10^{-6}$ & $6.76 \times 10^{-7}$ & $1.35 \times 10^{-1}$ \\
0.6 & $1.11 \times 10^{-9}$ & $6.89 \times 10^{-6}$ & $4.56 \times 10^{-5}$ & $7.20 \times 10^{-5}$ \\
0.7 & $7.88 \times 10^{-10}$ & $6.19 \times 10^{-6}$ & $7.05 \times 10^{-5}$ & $1.27 \times 10^{-1}$ \\
0.8 & $3.20 \times 10^{-10}$ & $5.83 \times 10^{-6}$ & $4.41 \times 10^{-5}$ & $7.48 \times 10^{-2}$ \\
0.9 & $2.34 \times 10^{-10}$ & $6.61 \times 10^{-6}$ & & $3.84 \times 10^{-2}$ \\
\hline
\end{tabular}

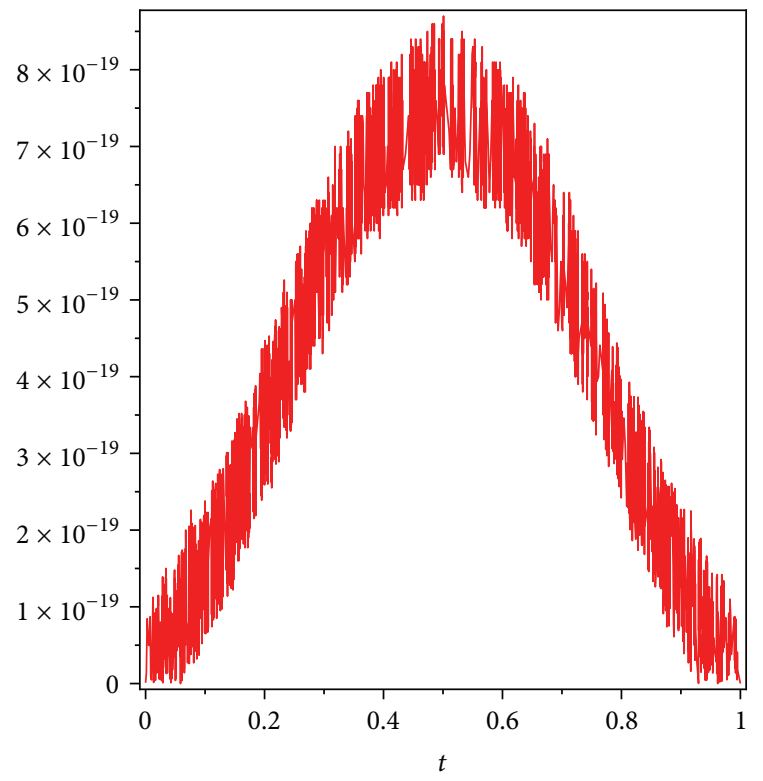

Figure 2: Plot of absolute errors for $\lambda=1$.

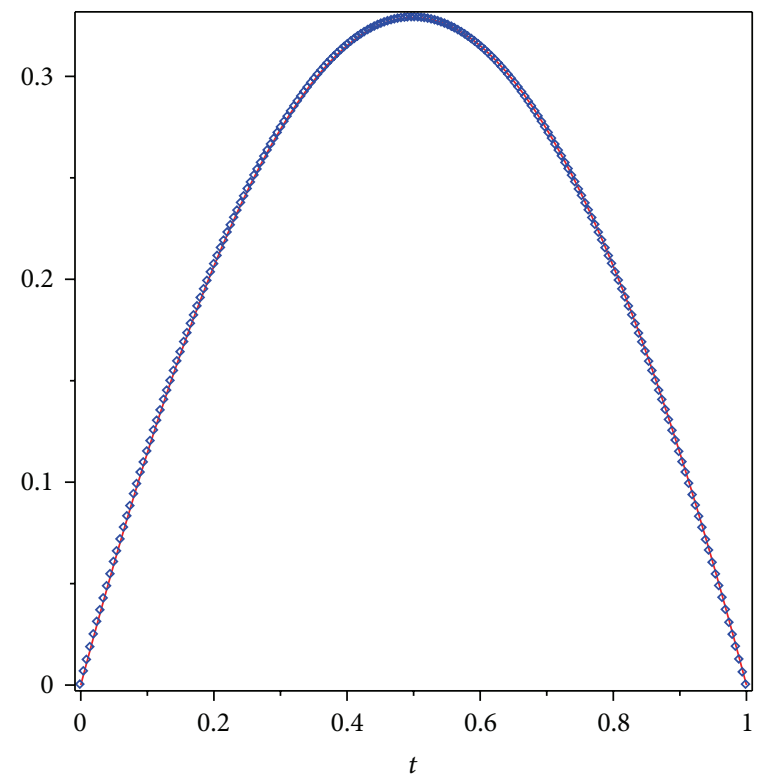

Figure 3: Plot of exact and approximated solutions for $\lambda=2$. method is reducing the given problems to those of solving a system of algebraic equations, thus greatly simplifying the problems. Sparseness of the coefficients matrix of algebraic equations makes it computationally efficient to solve these problems using the current method. It is also seen that increasing the number of subintervals or the number of collocation points in subintervals results in improving the accuracy. Numerical results confirm that our method is much better than other reported ones in the literature in the sense of accuracy and efficiency. According to Tables 1-4, our 
TABLE 8: Obtained maximum absolute errors.

\begin{tabular}{lcccccc}
\hline & $M=8, k=1$ & $M=8, k=2$ & $M=8, k=3$ & $M=10, k=1$ & $M=10, k=2$ & $M=10, k=3$ \\
\hline$\lambda=1$ & $2.5 \times 10^{-10}$ & $5.0 \times 10^{-12}$ & $3.0 \times 10^{-15}$ & $1.2 \times 10^{-12}$ & $8.0 \times 10^{-16}$ & $8.0 \times 10^{-19}$ \\
$\lambda=2$ & $1.8 \times 10^{-8}$ & $1.5 \times 10^{-10}$ & $1.6 \times 10^{-13}$ & $1.5 \times 10^{-10}$ & $7.0 \times 10^{-14}$ & $6.9 \times 10^{-17}$ \\
$\lambda=3.51$ & $3.0 \times 10^{-5}$ & $1.8 \times 10^{-8}$ & $1.0 \times 10^{-9}$ & $4.0 \times 10^{-7}$ & $2.0 \times 10^{-9}$ & $1.2 \times 10^{-9}$ \\
\hline
\end{tabular}

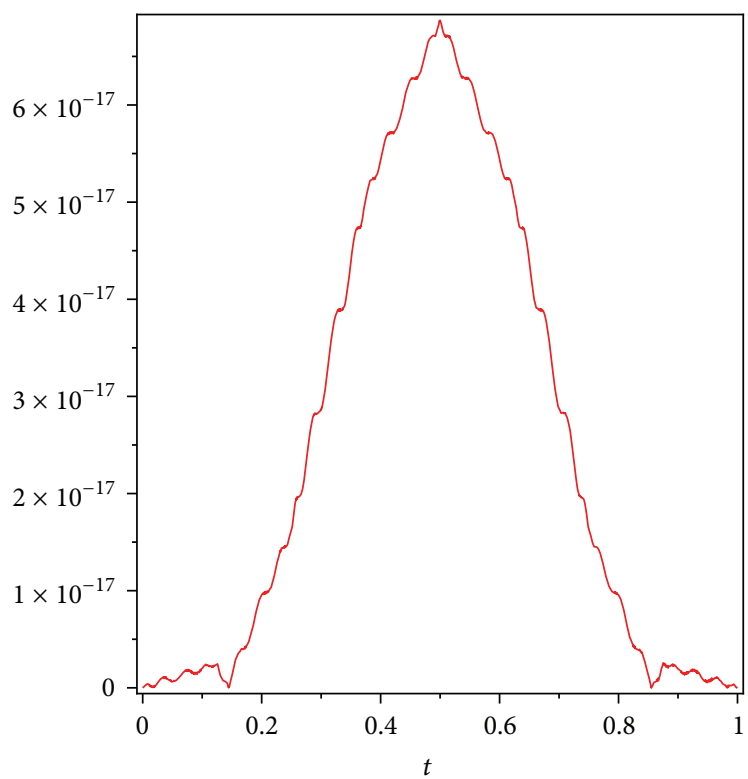

FIGURE 4: Plot of absolute errors for $\lambda=2$.

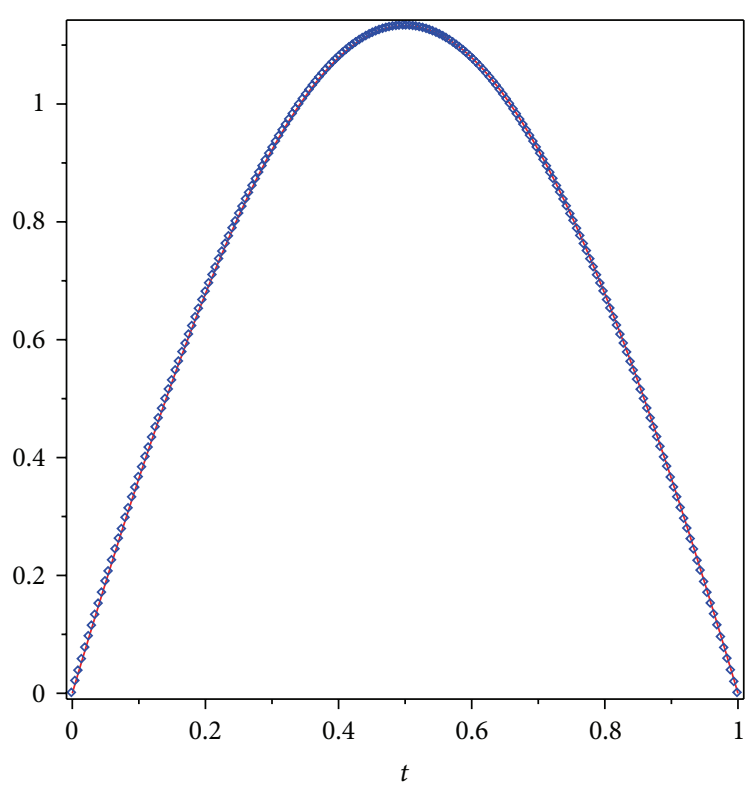

FIgURE 5: Plot of exact and approximated solutions for $\lambda=3.51$.

approach is applicable to solve Troesch's problem especially when $\lambda$ is large while some other methods fail to do so. As shown in Tables 5-8, we can obtain the results for Bratu's problem only by using a small number of Chebyshev wavelet basis functions. When $\lambda$ is close to the critical value $\lambda_{c}$,

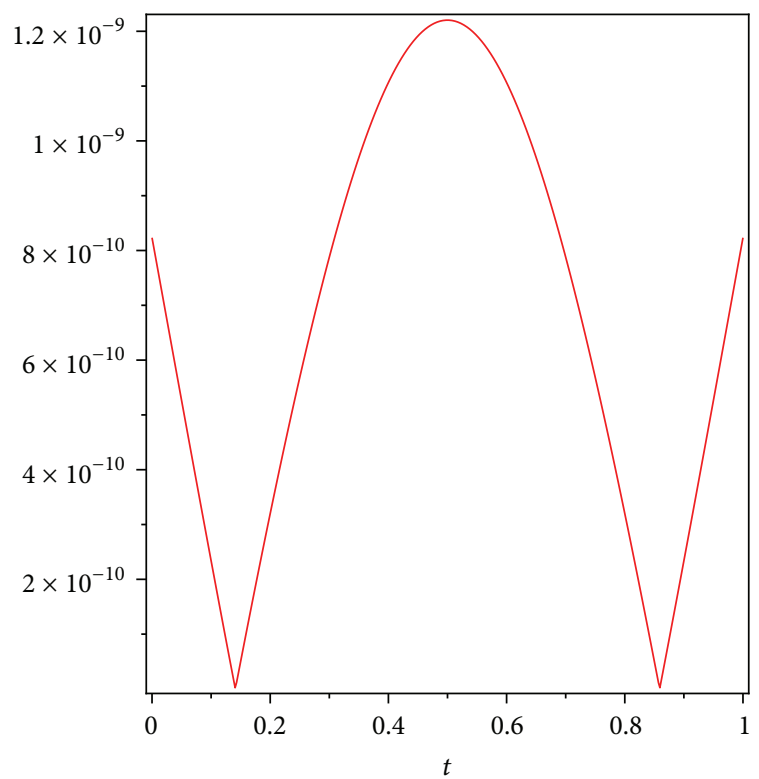

FIGURE 6: Plot of absolute errors for $\lambda=3.51$.

the wavelet analysis method was also accurate to the ninth order, whereas other methods especially the B-spline method yielded poorer results.

\section{Acknowledgment}

The authors gratefully acknowledge that this research was partially supported by the Universiti Putra Malaysia under the ERGS Grant Scheme having project number 5527068.

\section{References}

[1] M. Abramowitz and I. Stegun, Handbook of Mathematical Functions, Dover, New York, NY, USA, 1972.

[2] A. Erdelyi, W. Magnus, F. Oberhettinger, and F. G. Tricomi, Higher Transcendental Functions, vol. 2, McGraw-Hill, New York, NY, USA, 1953.

[3] S. M. Roberts and J. S. Shipman, "On the closed form solution of Troesch's problem," Journal of Computational Physics, vol. 21, no. 3, pp. 291-304, 1976.

[4] E. S. Weibel, "On the confinement of a plasma by magnetostatic fields," Physics of Fluids, vol. 2, no. 1, pp. 52-56, 1959.

[5] B. A. Troesch, "Intrinsic difficulties in the numerical solution of a boundary value problem," Internal Report 142, TRW Inc., Redondo Beach, Calif, USA, 1960.

[6] E. Deeba, S. A. Khuri, and S. Xie, "An algorithm for solving boundary value problems," Journal of Computational Physics, vol. 159, no. 2, pp. 125-138, 2000. 
[7] X. Feng, L. Mei, and G. He, "An efficient algorithm for solving Troesch's problem," Applied Mathematics and Computation, vol. 189, no. 1, pp. 500-507, 2007.

[8] S. Momani, S. Abuasad, and Z. Odibat, "Variational iteration method for solving nonlinear boundary value problems," Applied Mathematics and Computation, vol. 183, no. 2, pp. 1351$1358,2006$.

[9] S. A. Khuri, "A numerical algorithm for solving Troesch's problem," International Journal of Computer Mathematics, vol. 80, no. 4, pp. 493-498, 2003.

[10] S. A. Khuri and A. Sayfy, "Troesch's problem: a B-spline collocation approach," Mathematical and Computer Modelling, vol. 54, no. 9-10, pp. 1907-1918, 2011.

[11] M. Zarebnia and M. Sajjadian, "The sinc-Galerkin method for solving Troesch's problem," Mathematical and Computer Modelling, vol. 56, no. 9-10, pp. 218-228, 2012.

[12] S.-H. Chang and I.-L. Chang, "A new algorithm for calculating one-dimensional differential transform of nonlinear functions," Applied Mathematics and Computation, vol. 195, no. 2, pp. 799805, 2008.

[13] S.-H. Chang, "A variational iteration method for solving Troesch's problem," Journal of Computational and Applied Mathematics, vol. 234, no. 10, pp. 3043-3047, 2010.

[14] M. R. Scott, "On the conversion of boundary-value problems into stable initial-value problems via several invariant imbedding algorithms," in Numerical Solutions of Boundary-Value Problems for Ordinary Differential Equations, A. K. Aziz, Ed., pp. 89-146, Academic Press, New York, NY, USA, 1975.

[15] S. M. Roberts and J. S. Shipman, "Solution of Troesch's twopoint boundary value problem by a combination of techniques," Journal of Computational Physics, vol. 10, pp. 232-241, 1972.

[16] L. Bougoffa and M. A. Al-khadhi, "New explicit solutions for Troesch's boundary value problem," Applied Mathematics \& Information Sciences, vol. 3, no. 1, pp. 89-96, 2009.

[17] S. H. Mirmoradi, I. Hosseinpour, S. Ghanbarpour, and A. Barari, "Application of an approximate analytical method to nonlinear Troesch's problem," Applied Mathematical Sciences, vol. 3, no. 29-32, pp. 1579-1585, 2009.

[18] H. N. Hassan and M. A. El-Tawil, "An efficient analytic approach for solving two-point nonlinear boundary value problems by homotopy analysis method," Mathematical Methods in the Applied Sciences, vol. 34, no. 8, pp. 977-989, 2011.

[19] S. T. Mohyud-Din, "Solution of Troesch's problem using He's polynomials," Revista de la Unión Matemática Argentina, vol. 52, no. 1, pp. 143-148, 2011.

[20] D. D. Joseph and T. S. Lundgren, "Quasilinear Dirichlet problems driven by positive sources," Archive for Rational Mechanics and Analysis, vol. 49, pp. 241-269, 1973.

[21] J. P. Boyd, "Chebyshev polynomial expansions for simultaneous approximation of two branches of a function with application to the one-dimensional Bratu equation," Applied Mathematics and Computation, vol. 143, no. 2-3, pp. 189-200, 2003.

[22] Y. A. S. Aregbesola, "Numerical solution of Bratu problem using the method of weighted residual," Electronic Journal of Southern African Mathematical Sciences, vol. 3, no. 1, pp. 1-7, 2003.

[23] A.-M. Wazwaz, "Adomian decomposition method for a reliable treatment of the Bratu-type equations," Applied Mathematics and Computation, vol. 166, no. 3, pp. 652-663, 2005.

[24] M. I. Syam and A. Hamdan, "An efficient method for solving Bratu equations," Applied Mathematics and Computation, vol. 176, no. 2, pp. 704-713, 2006.
[25] R. Buckmire, "Application of a Mickens finite-difference scheme to the cylindrical Bratu-Gelfand problem," Numerical Methods for Partial Differential Equations, vol. 20, no. 3, pp. 327-337, 2004.

[26] J. S. McGough, "Numerical continuation and the Gelfand problem," Applied Mathematics and Computation, vol. 89, no. 1-3, pp. 225-239, 1998.

[27] A. S. Mounim and B. M. de Dormale, "From the fitting techniques to accurate schemes for the Liouville-Bratu-Gelfand problem," Numerical Methods for Partial Differential Equations, vol. 22, no. 4, pp. 761-775, 2006.

[28] S. Li and S.-J. Liao, "An analytic approach to solve multiple solutions of a strongly nonlinear problem," Applied Mathematics and Computation, vol. 169, no. 2, pp. 854-865, 2005.

[29] S. Liao and Y. Tan, "A general approach to obtain series solutions of nonlinear differential equations," Studies in Applied Mathematics, vol. 119, no. 4, pp. 297-354, 2007.

[30] S. A. Khuri, "A new approach to Bratu's problem," Applied Mathematics and Computation, vol. 147, no. 1, pp. 131-136, 2004.

[31] R. Jalilian, "Non-polynomial spline method for solving Bratu's problem," Computer Physics Communications, vol. 181, no. 11, pp. 1868-1872, 2010

[32] H. Caglar, N. Caglar, M. Özer, A. Valarıstos, and A. N. Anagnostopoulos, "B-spline method for solving Bratu's problem," International Journal of Computer Mathematics, vol. 87, no. 8, pp. 1885-1891, 2010.

[33] I. H. Abdel-Halim Hassan and V. S. Ertürk, "Applying differential transformation method to the one-dimensional planar Bratu problem," International Journal of Contemporary Mathematical Sciences, vol. 2, no. 29-32, pp. 1493-1504, 2007.

[34] B. Batiha, "Numerical solution of Bratu-type equations by the variational iteration method," Hacettepe Journal of Mathematics and Statistics, vol. 39, no. 1, pp. 23-29, 2010.

[35] S. Abbasbandy, M. S. Hashemi, and C.-S. Liu, "The Lie-group shooting method for solving the Bratu equation," Communications in Nonlinear Science and Numerical Simulation, vol. 16, no. 11, pp. 4238-4249, 2011.

[36] M. Misiti, Y. Misiti, G. Oppenheim, and J. -M Poggi, Wavelets Toolbox Users Guide. The MathWorks, Wavelet Toolbox, for use with Matlab, 2000.

[37] S. Gh. Hosseini and F. Mohammadi, "A new operational matrix of derivative for Chebyshev wavelets and its applications in solving ordinary differential equations with non analytic solution," Applied Mathematical Sciences, vol. 5, no. 49-52, pp. 2537-2548, 2011.

[38] G. B. Folland, Real Analysis: Modern Techniques and Their Applications, Pure and Applied Mathematics, John Wiley \& Sons Inc., New York, NY, USA, Second edition, 1999.

[39] I. Daubechies, Ten Lectures on Wavelets, vol. 61 of CBMSNSF Regional Conference Series in Applied Mathematics, SIAM, Philadelphia, Pa, USA, 1992. 


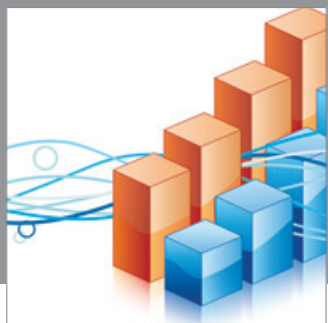

Advances in

Operations Research

mansans

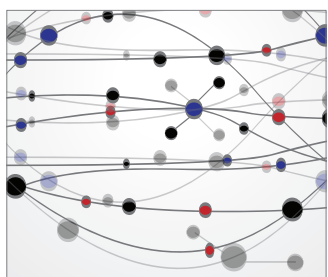

The Scientific World Journal
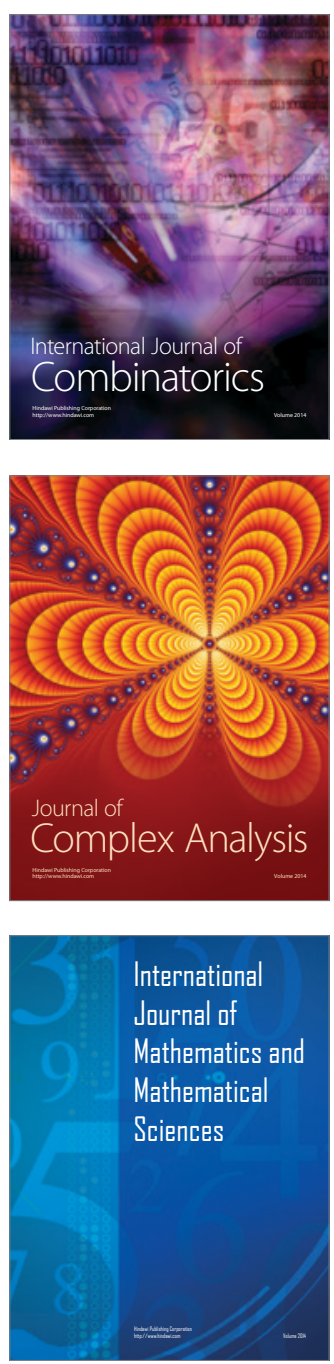
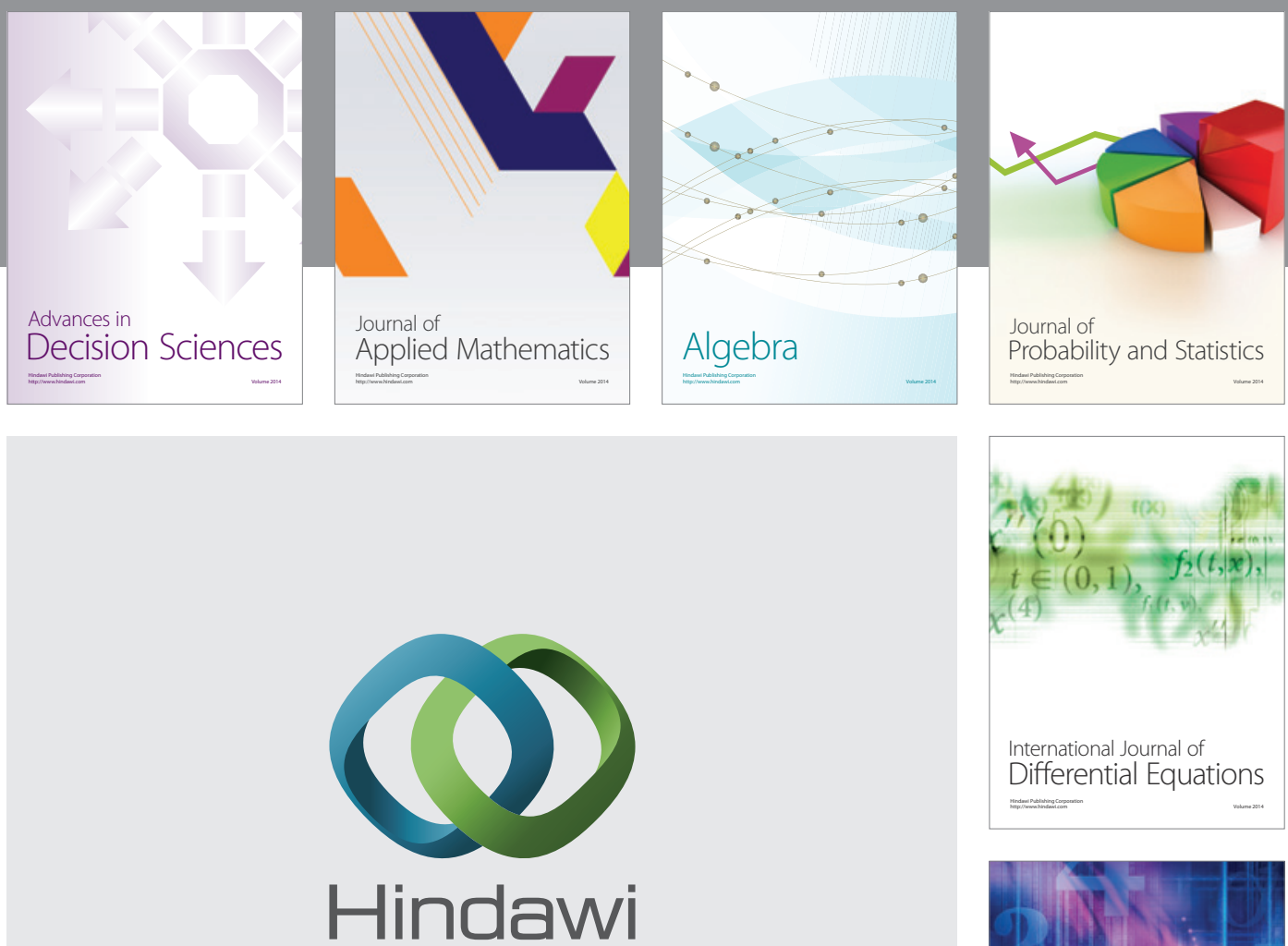

Submit your manuscripts at http://www.hindawi.com
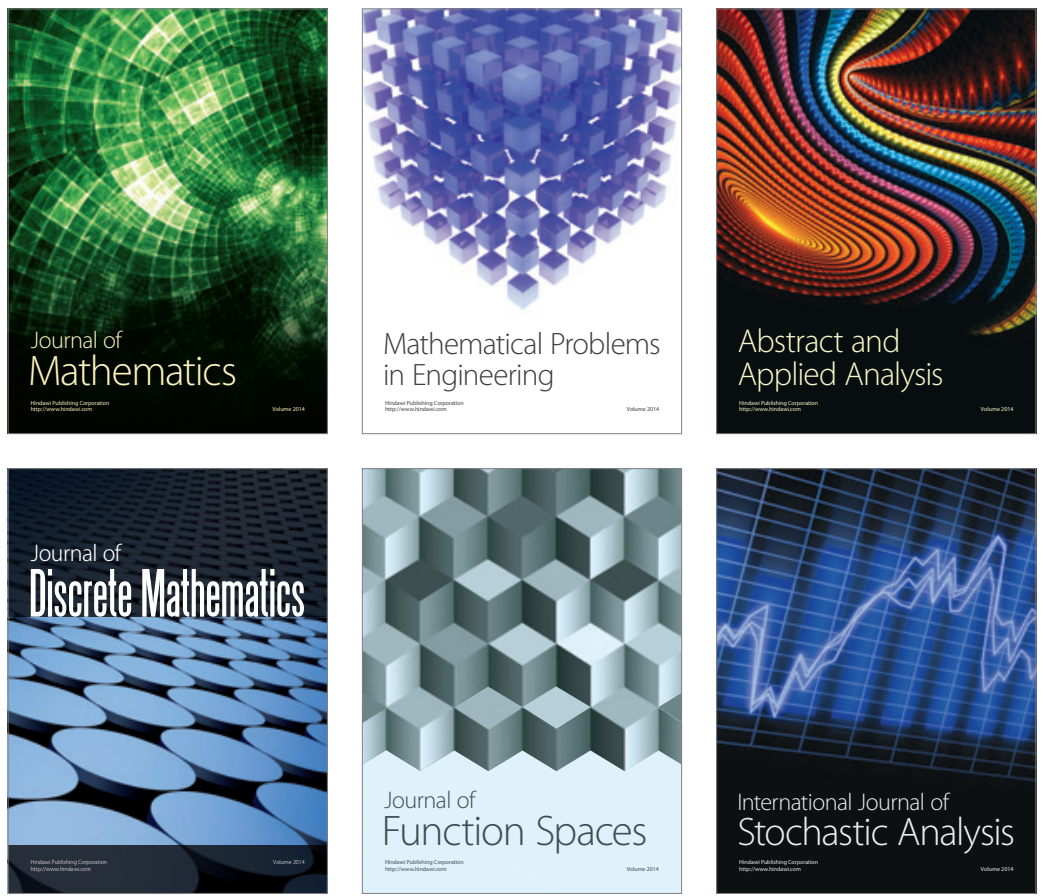

Journal of

Function Spaces

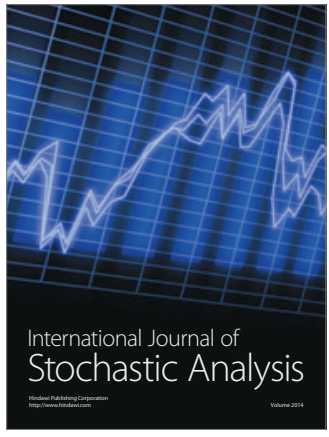

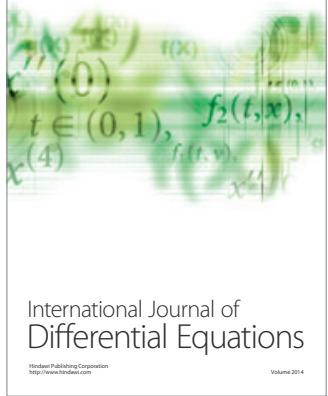
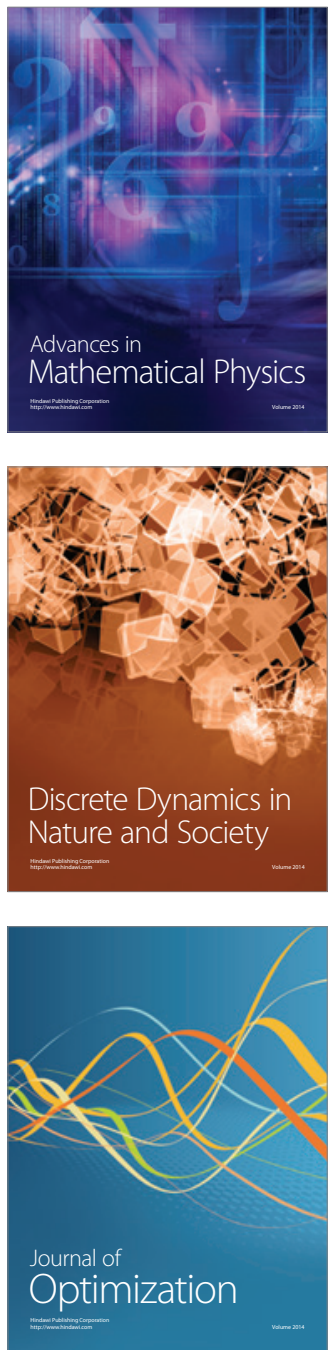